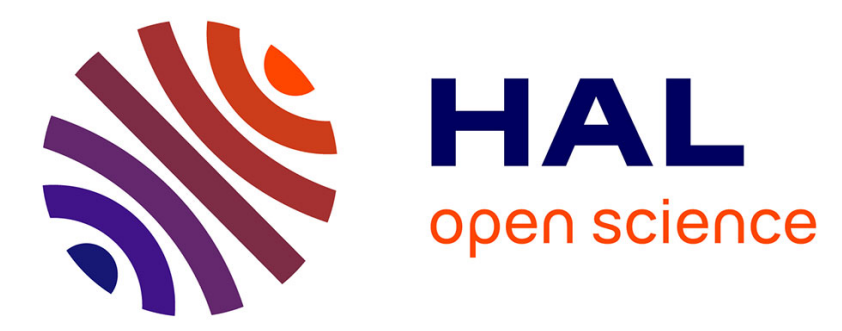

\title{
Real time ultrasound assessment of contact progress between food gels and tongue mimicking surfaces during a compression
}

Mathieu Mantelet, Rohit Srivastava, Frederic Restagno, Isabelle Souchon, Vincent Mathieu

\section{To cite this version:}

Mathieu Mantelet, Rohit Srivastava, Frederic Restagno, Isabelle Souchon, Vincent Mathieu. Real time ultrasound assessment of contact progress between food gels and tongue mimicking surfaces during a compression. Food Hydrocolloids, 2020. hal-02992136

\section{HAL Id: hal-02992136 \\ https://hal.science/hal-02992136}

Submitted on 6 Nov 2020

HAL is a multi-disciplinary open access archive for the deposit and dissemination of scientific research documents, whether they are published or not. The documents may come from teaching and research institutions in France or abroad, or from public or private research centers.
L'archive ouverte pluridisciplinaire HAL, est destinée au dépôt et à la diffusion de documents scientifiques de niveau recherche, publiés ou non, émanant des établissements d'enseignement et de recherche français ou étrangers, des laboratoires publics ou privés. 

5

\title{
Real time ultrasound assessment of contact
} progress between food gels and tongue mimicking surfaces during a compression

\author{
Mathieu MANTELET ${ }^{a}$, Rohit SRIVASTAVA ${ }^{a}$, Frédéric RESTAGNO ${ }^{b}$, Isabelle SOUCHON ${ }^{c}$ and Vincent \\ MATHIEU \\ a Université Paris-Saclay, INRAE, AgroParisTech, UMR SayFood, F-78850, Thiverval-Grignon, France \\ b Université Paris-Saclay, CNRS, Laboratoire de physique des solides, 91400 Orsay, France \\ c INRAE, Avignon Université, UMR408 SQPOV, F-84000 Avignon, France
}

\section{Submitted to Food Hydrocolloids}

Declarations of interest: none. . 7

Corresponding author:

9 Vincent Mathieu

Paris-Saclay Food and Bioproduct Engineering Research Unit (SayFood UMR 782)

1 Joint Research Unit INRAE AgroParisTech

2 Bâtiment CBAl

3 1, avenue Lucien Brétignières

478850 Thiverval-Grignon, France

5 Mail: vincent.mathieu@inrae.fr

6 Phone: +33(0)130816813

7 Fax: +33(0)1 30815597 
An in vitro study was conducted to further explore the potential of an emerging ultrasound method for monitoring in real time the progress of the contact between gels with different physical properties and tongue mimicking surfaces (TMSs) during uniaxial compressions. A 1-MHz ultrasound transducer was used to measure the apparent reflection coefficient $\left(R^{*}\right)$ of the interface between TMSs (of varied roughness) and gels (agar and/or gelatin). The patterns of $R^{*}$ clearly depended on the gels capacity to deform and mould the asperities of the TMSs during compression. Rough TMSs induced a significant decline of $R^{*}$ values, demonstrating an increase of the fraction of the TMSs in direct contact with the gels. Rigidity, fracture properties or adhesion energy of gels influenced the kinetics of contact progress and associated patterns of $R^{*}$ variations. Moreover, the presence of a thick lubrication film between the gels and the TMSs promoted the transmission of ultrasound waves (acting as a coupling agent) and led to decreasing values of $R^{*}$. Such phenomena were observed both when depositing a water lubrication film of controlled thickness on the TMSs (to mimic salivary film) and when considering gels with high water release capacities. The study confirms the potential of ultrasound methods for exploring physical phenomena related to interactions between food and perceptions during food oral processing. 


\section{Introduction}

\subsection{Role of the tongue in food oral processing and texture perceptions}

Food oral processing plays a major role in consumers' appreciation of food as it encompasses dynamic, multi scale and multi-sensory perceptions. Food is subjected to multiple biomechanical and chemical processes in oral cavity (Hutchings \& Lillford, 1988; Stokes et al., 2013). It is chewed by the teeth, moistened by the saliva, mixed and sheared by the action of the tongue, the palate and the cheeks, adjusted to suitable oral temperature, so that it can be transformed into a bolus ready to be swallowed in safe and comfortable conditions (Hiiemae, 2004). Each one of these actions leads to the stimulation of sensory receptors that make it possible to monitor the changes in ingested food during oral processing.

Even if the consumer needs to adapt his or her strategy of oral processing to the wide diversity of food structures, the manipulation of food between the tongue and the palate is a step required for every food products and at every moment of oral processing. The dynamic physical interactions at tongue-food interface give rise to the activation of various mechanoreceptors located in tongue, and which highly contribute to the perception of food texture (Bukowska et al., 2010). Since the tongue plays such a crucial part in the sensory experience of the consumer, a better understanding of texture perceptions resulting from tongue-food-palate interactions may provide important benefits for the design of new foods with improved sensory properties.

Moreover, the tongue also makes it possible to evaluate in real time the changes of the mechanical status of the bolus in order to decide the most appropriate moment for triggering a safe swallowing sequence (J. Chen, 2009; Hutchings \& Lillford, 1988). Precisely, understanding the mechanisms of texture perceptions between the tongue and the palate could be helpful for the design of an alternative food supply for specific populations facing swallowing disorders (dysphagia). The response to this challenge requires the unraveling of the dynamic structural and mechanical 
transformations undergone at tongue-food interface during oral processing (Foegeding et al., 2017; Hutchings \& Lillford, 1988; Panouillé et al., 2016; Szczesniak, 2002).

\subsection{Need for in-situ and dynamic instrumental characterizations}

The development of technical approaches is essential for exploring the physical phenomena involved in texture perceptions. Numerous studies have attempted to mimic some specific sequences of food oral processing with mechanical testing or rheological approaches (J. S. Chen \& Stokes, 2012). Even though these technics are suitable for the characterization of the transformations in the bulk of food, they do not make it possible to comprehend the physical information related to the surface properties, which are critical for tactile mechano-sensations. Tribology has also been proposed as an effective tool to describe, at the end of oral processing or right after swallowing, the friction mechanisms due to the interaction between the surface of the tongue and food bolus (Stokes et al., 2013). However, from the introduction in the oral cavity to swallowing, food undergoes major transformations, in such a way that oral processing goes from rheology to tribology dominant phases. As a consequence, the development of in-situ and non-destructive methods suitable for the continuous evaluation of the different phases of oral processing represents a critical research gap (Foegeding et al., 2017).

\subsection{The potential of ultrasound techniques}

Ultrasound (US) methods could help to address this challenge. US imaging has already been used in the oral cavity for various applications, including the study of food bolus transportation during swallowing (de Wijk et al., 2011; Galén \& Jost-Brinkmann, 2010; Peng et al., 2000), of tongue shape and motions during speech (Stone, 2005), of functional disorders such as dysphagia (Hsiao et al., 2012; Kim \& Kim, 2012; Rommel \& Hamdy, 2016) or also of obstructive sleep apnea events (J.-W. Chen et al., 2014; Chien et al., 2017; Weng et al., 2017). The wide diversity of these fields of application confirms the capability of US methods for the real-time exploration of dynamic mechanisms in oral cavity. 

interfaces they propagate through. For instance, ultrasound technics have thus been proposed to characterize food composition, structure, or transformation processes in various products such as meat, fruits, vegetables, emulsions or gels (Awad et al., 2012).

A first exploratory study has investigated the variations of the apparent reflection coefficient (referred to as $R^{*}$ hereafter) of ultrasound waves at the interface between non-deformable tongue mimicking surfaces (TMS hereafter) and agar/gelatin mixed gels with various physical properties (Mantelet et al., 2020). The results have shown that the US method has a high potential for the characterization of the capability of food gels to mold themselves into the surface asperities. The properties of both TMSs (roughness, lubrication) and food (Young's modulus, syneresis) were shown to have an impact on $R^{*}$ values. In static conditions, the apparent reflection coefficient was significantly larger in the following situations: (i) tongue asperities were high and dense; (ii) lubrication levels were low; and (iii) gels were less rigid. The apparent reflection coefficient conveyed the ability of food gels to mold themselves to surface asperities or to form a coupling film of liquid at the interface. These preliminary experiments were conducted in static conditions, with the aim to mimic the initial contact between food and the surface of the tongue. However, no mechanical load was applied to the food samples and it is now required to go further and investigate the behavior of the US response of the system during the break down of food.

For that purpose, the present study aims at investigating the evolution of $R^{*}$ during a uni-axial

114 compression mimicking the initial step of food oral processing. The same TMSs and model gels as those 115 considered in the first study have been used to explore the impact of their respective properties on 116 the variations of $R^{*}$ (Mantelet et al., 2020).

\subsection{Model foods}



following the same protocol (Mantelet et al., 2020). These gels are composed of agar (HP700IFG, Kalys, Bernin, France) and gelatin (Bloom 250 PS 8/3, Rousselot, Gent, Belgium), sucrose (Daddy, Cristalco, Paris, France) and water. For one of the eight samples Tween 20 (CAS: 9005-64-5 FG, Sigma-Aldrich, St Louis, MO, USA) emulsifier was used to alter sample's surface wettability. All the gels underwent identical thermal treatments, so that to dissolve sucrose, agar and gelatin at different heating steps. Firstly, solutions of water with sucrose were prepared and stirred for $30 \mathrm{~min}$ at room temperature ensuring the complete dissolution of agar. Thirdly, solutions were cooled and stabilized for 5 min to $60^{\circ} \mathrm{C}$. Lastly, gelatin powder was added (for gels with gelatin) and all solutions were stirred for $20 \mathrm{~min}$ at $60^{\circ} \mathrm{C}$. Solutions at $60^{\circ} \mathrm{C}$ were then poured in polyethylene cylindrical molds and left at $19{ }^{\circ} \mathrm{C}$ during $15 \mathrm{~h}-18 \mathrm{~h}$. The gels cylinders of $30 \mathrm{~mm}$ in diameter and $10 \mathrm{~mm}$ in height were individually unmolded at the last moment before the experiments. The gels can be classified in two main categories: $A g_{0.3}$, $A g_{1.8}$ and $A g_{1} G e_{0.75}$ are agar dominant samples, whereas $G e_{3.5}, A g_{0.3} G e_{3.5}, A g_{0.7} G e_{5.85}, G e_{7}$ and $G e_{7} T$ are gelatin dominant samples. It is important to note that $\mathrm{Ge}_{7} T$ differs from $\mathrm{Ge}_{7}$ only by the presence of Tween 20. Introducing this emulsifier led a to a decrease of the contact angle (from 40 to $0^{\circ}$ ) (Mantelet et al., 2019). The underlying objective was to investigate whether the wettability of the gels is likely to influence the patterns of due to differences in water film spreading at the interface between the TMS and the food. Table 1 summarizes, for each of the eight gels considered here, the type of dominant polymer, the composition and the Young's modulus obtained from previous characterizations (Mantelet et al., 2020).

\subsection{Tongue mimicking surfaces}

As in our previous study, three cylinders of PVC (polyvinyl chloride) were used as TMSs

142 (Mantelet et al., 2020). The diameter of the PVC cylinders $(50 \mathrm{~mm}$ ) was kept larger than that of model 
144 chosen to be in accordance with the acoustic properties of PVC (Mantelet et al., 2020). Three types of

145 TMSs were used in this study, with different surface roughness characteristics imprinted by sand 146 papers during the course of cylinder moulding. Referred to as $R_{0} b_{0}, R_{1} B_{1}$ and $R_{1} b_{2}$, the TMSs differ 147 specifically by their arithmetical mean height $R a$ (mean \pm standard deviation of the height of the 148 asperities, respectively equal to $2.5 \pm 0.1,58.2 \pm 5.3$ and $52 \pm 11.2 \mu \mathrm{m}$ ) and by their correlation length $\beta$ 149 (related to the density of the asperities and respectively, with mean \pm standard deviation equal to $15035 \pm 0.4,206 \pm 4$ and $243 \pm 12 \mu \mathrm{m}$ ). Both the above mentioned parameters were obtained through 151 profilometry measurements and were found to be reproducible for each TMS production (Mantelet et 152 al., 2020). The mean height of the asperities on the surface of real tongues has been estimated to 153 range from 40 and $100 \mu \mathrm{m}$, showing that $R_{1} B_{1}$ and $R_{1} B_{2}$ displayed physiological relevant values (Uemori 154 et al., 2012). Conversely, $R_{0} B_{0}$ can be considered as smooth when compared to $R_{1} B_{1}, R_{1} B_{2}$, or to real 155 tongues. This TMS can thus be used as a control, making it possible to discuss the impact of surface 156 roughness with negligible effects of contact progress between the gels and the TMSs.

For the sake of simplicity, water was used as a lubricant to mimic the lubrication of the tongue

158 surface by saliva. Four lubrication levels were considered, referred to as dry, low, medium and high 159 (Mantelet et al., 2020). Dry (absence of lubricant) and high (water film of $1 \mathrm{~mm}$ thick) levels were 160 voluntarily chosen to compare highly contrasting conditions. The high lubrication level is much higher 161 than orders of magnitude of salivary film thickness. This condition made it possible to ensure that 162 water was the only medium present at the interface between the food and the TMS. The underlying 163 objective was to investigate whether the wettability of the gels is likely to influence the ultrasound 164 response at the interface between the TMS and food, due to potential differences in water film 165 spreading at the interface. Low and medium levels correspond to 15 and $40 \mu \mathrm{m}$ thick water films 166 respectively, deposited on the TMS with a spray in a controlled and reproducible way.

\subsection{Compression protocol}


169 was used to simulate the tongue/palate compression (see Figure 1). The TMS was mounted on the 170 base of the apparatus, while a circular aluminum probe (diameter $40 \mathrm{~mm}$ ) was used to play the role of 171 the hard palate. The food samples underwent $80 \%$ uni-axial compressions at $10 \mathrm{~mm} \cdot \mathrm{s}^{-1}$, which was the highest value of velocity attainable with the set-up. The compression protocol was composed of different steps. conditions described above. The food sample to be tested was unmoulded and deposited on the TMS right before the experiment, to prevent any syneresis, drying and sagging over time. Then, the mimicking palate was put in contact with the food sample and the following compression sequence was applied: a static holding step of $3 \mathrm{~s}$ at $0 \%$ strain, followed by a compression step of $80 \%$ at 10 $\mathrm{mm} \cdot \mathrm{s}^{-1}$, and finally a second holding step of $3 \mathrm{~s}$. gel on a TMS of a given roughness and with a given lubrication level).

\subsection{Ultrasound measurements}

The ultrasonic measurements aim at monitoring the evolution of $R^{*}$ during the compression of the different food gels on the bio-mimicking set up described above. The device and the procedure used for ultrasound measurements is the same as in our previous paper (Mantelet et al., 2020).

\subsubsection{Ultrasonic device}

The device was composed of a mono-element piezoelectric transducer (V103RM, Olympus, Shinjuku, Tokyo, Japan) with a central frequency of $900 \mathrm{kHz}$ (longitudinal waves). The US transducer 
193 signal (500 ns width, 80 Volt amplitude) for the emission of the US pulse, and which digitalized the

194 radio frequency $(r f$ ) signals corresponding to the pulse echo response of the system (12-bit quantification, $100 \mathrm{MHz}$ sampling rate, $38 \mathrm{~dB}$ gain). The acquisition of the $r f$ signals was done in real time during the compressing tests using a dedicated user-interface developed with LabVIEW ${ }^{\circledR}$ (LabVIEW, National Instrument, Austin, Texas, USA). The pulse recurrence frequency was of around 90 $\mathrm{Hz}$. The typical number of signals acquired during a compression was of around 70.

\subsubsection{Signal processing}

The sets of $r f$ signals obtained for each compression test were processed with MATLAB ${ }^{\circledast}$ (The MathWorks, Natick, Massachussetts, USA). $R^{*}$ was calculated for each individual $r f$ signal, following the protocol extensively described in our previous study (Mantelet et al., 2020). To summarize it briefly, the first step consisted of high-frequency noise reduction with a low-pass filter (15 MHz cutoff frequency) applied to the Fast Fourier Transform (FFT) of the $r f$ signals. The $r f$ signals were composed of two main echoes (see Figure 1.b): $E_{0}$ corresponds to the acoustic energy reflected at the interface between the TMS and the food gel, whereas $E_{1}$ is the response of the interface between the food gel and the palate. During the uniaxial compression (see Figure 1.c), the time of flight of $E_{1}$ decreases and tends to get closer to $E_{0}$. The present study focuses on $E_{0}$ but some undesired artefacts may occur by the end of the compression due to overlapping between $E_{0}$ and $E_{1}$. Time intervals of interest (4 $\mu \mathrm{s}$ width) centered on the maximum of the modulus of the Hilbert transform of echo $E_{0}$ (see Figure 1.b) were determined to track the echo of the TMS. The amplitude $A_{0}$, defined as the modulus of the FFT of these temporal windows at $900 \mathrm{kHz}$, was then calculated for each $r f$ signal. Finally, $R^{*}$ was obtained following:

$$
R^{*}(\varepsilon)=\frac{A_{0}(\varepsilon)}{A_{0, \mathrm{ref}}}
$$


215 corresponding to each $r f$ signal and $A_{0, \text { ref }}$ is a reference value of the amplitude $A_{0}$ recorded before 216 lubricating the TMS and depositing the food sample.

$$
\Delta R^{*}(\varepsilon)=R^{*}(\varepsilon)-R^{*}(\varepsilon=0)
$$

In this way, it becomes easier to compare and analyze the amplitude of the variations of $R^{*}$ across the different experimental conditions.

\section{Results and discussion}

The results and discussion are structured in three distinct subsections, so that to discuss

successively the impact of (i) tongue roughness, (ii) food properties and (iii) tongue lubrication on the variations of $R^{*}$ during the compression of the food gels on TMSs. For all figures, the curves with identical colors correspond to replicates of identical conditions.

\subsection{Impact of surface roughness on $R^{*}$}

Figure 2.a represents the values of $R^{*}$ before the compression $(\varepsilon=0)$ of the gels $A g_{0.3}, G_{3.5}$, $G e_{7}$ on the three TMSs $R_{0} B_{0}, R_{1} B_{1}$, and $R_{1} B_{2}$ (in absence of lubrication). The three food gels selected here provide an overview of the various behaviors observed throughout the whole set of food samples. In the case of the smoothest TMS $\left(R_{0} B_{0}\right)$, similar values of $R^{*}$ were reported across the three food samples

231 (around $33 \%$ ). Contrarily, the presence of rough asperities on $R_{1} B_{1}$, and $R_{1} B_{2}$ led to higher and dispersed 232 values of $R^{*}$ across the three gels. The apparent reflection coefficient of US waves crossing the TMSs 233 depends on the gap of acoustic impedance $Z$ between the TMSs ( $Z \simeq 3.1$ MPa.s.m- 1 in PVC) and 234 surrounding media (Mantelet et al., 2019). In the present case, the surrounding media can be composed of air $(Z \simeq 420$ Pa.s.m-1), food gels ( $Z$ laying from 1.6 to 1.75 MPa.s.m- -1$)$ or of water $(Z \simeq$ 1.5 MPa.s.m-1) (Mantelet et al., 2019). Air has a negligible acoustic impedance when compared to PVC, 
237 food gels and water (the two latter being similar). The higher the gap of acoustic impendance at the 238 interface, the higher amplitude of US echo $E_{0}$ and subsequent value of $R^{*}$. If air fully covers a TMS, we 239 are in the case of a full reflection of the US wave and $R^{*}$ is close to $100 \%$. If contrarily the TMS is fully 240 imprinted with gels, $R^{*}$ has been show to range from 30 to $34 \%$, depending on the acoustic impedance 241 of the different gels (related to both mass density and compressibility) (Mantelet et al., 2019). When 242 a food sample is deposited on the TMS, a mix of air and gel in direct contact with the TMS is observed, 243 with intermediate values of $R^{*}$. Consequently, $R^{*}$ has been shown to be an indirect indicator of the 244 effective contact ratio between the food gel and the TMS (Mantelet et al., 2020). As a consequence, 245 Figure 2.a highlights that prior to the uni-axial compression, the surface $R_{0} b_{0}$ displayed a better 246 mechanical coupling with the food gels than the two other surfaces $\left(R_{1} B_{1}\right.$ and $\left.R_{1} B_{2}\right)$. Concerning $\Delta R^{*}$, an increase of $\Delta R^{*}$ during the compression of the gels means a spreading of 248 the surface of air in direct contact with the TMS. Conversely, a decrease of $\Delta R^{*}$ means that the surface 249 of gel or water in direct contact with the TMS tends to increase. Figure 2.b shows the variations of $\Delta R^{*}$ during a compression test for the same gels and TMSs (no lubrication). In the case of the smoothest TMS $\left(R_{0} B_{0}\right)$, no apparent variations of $\Delta R^{*}$ were reported during the compression of the different gels (blue curves in Figure 2.b), except in some cases at higher strains (higher than 70\%). As pointed out previously (Figure 1.c), the possibility of $E_{1}$ to overlap with $E_{0}$ at the end of the compression sequence might be responsible for this observed abnormal variation of amplitude at higher strains. Setting aside these undesired artefacts, the results suggest that the contact surface between the TMS $R_{0} B_{0}$ and food was not altered during the compression tests. For these three gels, $R^{*}$ was shown to range between 33 and $34 \%$ before the beginning of the compression (see Figure 2.a), suggesting a full contact between the TMS and the food. the compression (see Figure 2.b). These results suggest that the nature of the media in direct contact with the asperities of the TMSs varies when the food gel is under load. Interestingly, different trends 
were observed throughout the three gels in Figure 2.b. For gels $\mathrm{Ge}_{3.5}$ and $A g_{0.3}$, a slight increase of US

263 reflectivity was first observed, followed by a decreasing trend during the second portion of the

264 compression. However, for these two gels, no major differences were observed between $R_{1} B_{1}$ and $R_{1} B_{2}$.

265 The largest variations of $\Delta R^{*}$ were reported for $G e_{7}$, which subsequently decreased by $15 \%$ after $80 \%$ strain (see Figure 2. $b_{1}$ ). Interestingly, a faster decrease was observed on $R_{1} b_{1}$ at the beginning of the compression but later attaining the similar position as $R_{1} B_{2}$ by the end of compression.

contact between the food gel and the asperities of the TMSs. $R_{1} B_{1}$ and $R_{1} B_{2}$ have similar values of $R_{a}$ a higher density of surface asperities for $R_{1} B_{1}$ than for $R_{1} B_{2}$. As a consequence, the geometry of surface asperities may have an impact on the pattern of $\Delta R^{*}$ evolution during the compression. However, the gap of $R^{*}$ at the end of the compression was similar to what it was at the beginning (around $12 \%$ higher for $R_{1} B_{1}$ than for $R_{1} B_{2}$ ).

\subsection{Impact of food properties on $R^{*}$}

The food gels considered in the present study mainly differ by the composition of their agar/gelatin ratio. Due to phase separation mechanisms between agar and gelatin (Clark et al., 1983; McEvoy et al., 1985), the gels can be arranged in two groups: gelatin dominant gels consisting in a continuous phase of gelatin with, if appropriate, agar inclusions ( $\left.G e_{3.5}, A g_{0.3} G e_{3.5}, A g_{0.7} G e_{5.85}, G e_{7}\right)$, and conversely agar dominant gels composed of a continuous phase of agar with, if present, inclusions of 281 gelatin $\left(A g_{0.3}, A g_{1} G e_{0.75}, A g_{1.8}\right)$. Our previous study made it possible to show that for each type of samples, the higher the Young's modulus, the lower the capacity of the gels to mold the asperities of the TMS under the action of their own weight (without any imposed compression in that study), and the higher the apparent reflection coefficient $R^{*}$ (Mantelet et al., 2020). This is clearly related to the 
variations of $R^{*}$. As an example, $A g_{0.3}$ and $G e_{3.5}$ had similar values of rigidity but different values of $R^{*}$ 288 prior to their compression. The adhesive energy of the food gels on the surface of PVC may be a 289 contributing factor to the capacity of the gels to mold themselves into the asperities of the TMSs. Such properties are likely to vary according to the nature of the dominating polymer and to its level of concentration (Shull, 2002). The potential role of water release mechanisms (acting as a coupling media at the interface between the gel and the TMS and promoting the transmission of the US waves) was also considered to explain the different behaviours reported for the two groups of samples. For equal Young's moduli, the structure of agar dominant samples (double helix) could be more favorable to the release of free water (syneresis) than the one of gelatin dominant samples (triple helix). Such expelled water could act as a coupling media with the TMS and lead to lower values of $R^{*}$ for agar than for gelatin dominant samples (Santagiuliana et al., 2018). Once the water is released into the interface, the adhesion forces get disrupted, making syneresis the prominent factor.

Figure 3.a shows the variations of the applied force $F$ during the compression of the four gelatin dominant gels. For these same gels, Figure 3.b displays the variations of $\Delta R^{*}$ on the same deformation scale, while Figure 3.c represents the initial values of $R^{*}(\varepsilon=0)$ as a function of the Young's moduli of the samples. Similarly, Figure 4 displays the same parameters as in Figure 3, but for agar dominant gels: $F$ versus $\varepsilon$ in Figure 4.a, $\Delta R^{*}$ versus $\varepsilon$ in Figure 4.b, and $R^{*}(\varepsilon=0)$ versus $E$ in Figure 4.c. The results given in Figure 3 and Figure 4 were obtained with the TMS $R_{1} B_{1}$, and for the dry lubrication level. $\mathrm{Ge}_{3.5}$ was the gelatin dominant gel with the lowest level of concentration in biopolymer (and consequently with the lowest Young's modulus $-2.2 \mathrm{kPa}$ ). It also displayed the lowest level of variation 307 of $\Delta R^{*}$, suggesting that during the compression, the amount of direct contact between the asperities 308 of the TMS and the food did not deviate from the initial level. By adding only $0.3 \%$ of agar (in comparison with $\left.\mathrm{Ge}_{3.5}\right), \mathrm{Ag}_{0.3} \mathrm{Ge}_{3.5}$ displayed larger evolutions of $\Delta R^{*}$, with a slight increase during the first step of the compression, generally followed by a decrease, until recovering their initial values $\left(\Delta R^{*}\right.$ comprised between -2 and $+5 \%$ at the end of the compression). However, disparate behaviors were 
displayed across the four trials repeated for $\mathrm{Ag}_{0.3} \mathrm{Ge}_{3.5}$, suggesting that for this sample, the presence of

313 agar inclusions led to heterogeneous variations of the conditions of the contact with the TMS. The two

314 last gelatin dominant gels had a significantly higher Young's moduli (32.1 and $24.0 \mathrm{kPa}$ for $A g_{0.7} \mathrm{Ge}_{5.85}$

315 and $G e_{7}$ respectively). Both model food displayed declining trends of $\Delta R^{*}$ variations at the end of the compression (by 7 to 10 and 12 to $14 \%$ for $A g_{0.7} G e_{5.85}$ and $G e_{7}$ respectively). For $A g_{0.7} G e_{5.85}$, which was the most rigid, this decline of $\Delta R^{*}$ was faster at the beginning of the compression (between 0 and 15 $\%$ strain), followed by an increase at the very end of the compression (beyond $75 \%$ strain). The increase at the end coincides with the decrease reported on the force curve, which allows us to identify the fracture of the food sample. go through significant variations of $F$ and $\Delta R^{*}$ during the compression. Due to the low rigidity of $A g_{0.3}$ $323(E=4.2 \mathrm{kPa})$, the amplitude of the load transmitted to the interface during the compression may not 324 have been high enough so that to alter the nature of the media in direct contact with the TMS. 325 Moreover, the low values of $R^{*}$ reported before the beginning of the compression $\left(R^{*}(\varepsilon=0)=45.9 \%\right)$ indicate that even before the beginning of the compression, the coupling between the food and the TMS was already close to the threshold, providing a very little scope for further improvement. Although, distinct force-deformation curves were reported for more rigid gels like $A g_{1} G e_{0.75}$ and $A g_{1.8}$, with fracture occurring between 45 and $55 \%$. A rapid decrease of $\Delta R^{*}$ was observed in the first part of 330 the compression (before $40 \%$ deformation), all the more so when the Young's modulus of the sample 331 was high (between 10 and $18 \%$ for $A g_{1} G e_{0.75}$ while between 24 and $28 \%$ for $A g_{1.8}$ ). During this first 332 half of the compression, analogous patterns of $\Delta R^{*}$ were displayed inside the different food 333 references. These tendencies suggest that a significant increase of the amount of gels in contact with 334 the TMS occurred during the first part of the compression. After $40 \%$ strain, the $\Delta R^{*}$ curves exhibit 335 more individual behaviors, each showing an increase of $R^{*}$ that reflects relaxation mechanisms leading 336 to the withdrawal of the food sample from the asperities of the TMS. 
The experiments depicted in the first two parts of this discussion focused on investigating the behavior of $R^{*}$ in absence of lubrication at the interface between the TMS and the food. However, accounting for lubrication is also a critical issue for seeking to understand the role of the salivary film at this interface. In this feasibility study, a particular importance was granted to working with a stable and reproducible fluid, reason why water was preferred over real saliva. A recent work by our group displayed that in absence of mechanical loading (other than gravity), the lubrication of the TMS with water drastically affects the US response of the interface (Mantelet et al., 2020). compression. These two samples differ by the presence of Tween 20 in $G_{7} T$, an emulsifying agent used to modify surface tension properties (all by keeping the elastic properties unchanged in comparison with $\left.G_{7}\right)$. For the four lubrication conditions, Figure 5 shows the variations of $\Delta R^{*}$ as a function of strain for $G_{7}$ (Figure 5. $a_{1}$ and 5. $b_{1}$ ) and $G e_{7} T$ (Figure 5. $a_{2}$ and 5. $b_{2}$ ). Two distinct surface profiles were considered: $R_{0} b_{0}$ for Figures $5 . a_{1}$ and $5 . a_{2}, R_{1} b_{2}$ for Figures 5. $b_{1}$ and 5. $b_{2}$. lubrication conditions. On the same TMS $R_{0} B_{0}$, slightly higher decreases were displayed with $G e_{7} T$ : between 0 and $-1.2 \%$ for most of the tests, and as low as between -2 and $-4 \%$ in some isolated cases which were not related to a specific lubrication condition. For both samples, the initial value of $R^{*}$ was inferior to $40 \%$, suggesting that even before the beginning of the compression, the food gels were almost fully bonding the interface of the TMS. As a consequence, the margin available for improving the contact during the compression was low, explaining why low variations of $\Delta R^{*}$ were displayed. profile $R_{1} B_{2}$. 
$362 G e_{7}$ and by -13 to $-16 \%$ for $G e_{7} T$. These observations suggest a significant improvement of the acoustic 363 coupling at the interface between the TMS and the food. Interestingly, the initial value of $R^{*}$ was lower 364 for $G e_{7} T$ (mean \pm standard deviation equal to $63.6 \pm 0.4 \%$ ) than for $G_{7}$ (mean \pm standard deviation equal to $68.1 \pm 2.3 \%)$. As a consequence, $G e_{7} T$ displayed a higher capacity to fill the asperities of the TMS during the compression. A reason for these tendencies could be that Tween 20 may promote the adhesive properties of $\mathrm{Ge}_{7} T$, then altering the balance between adhesive and elastic forces that are jointly ruling the interaction between the food and the TMS. decrease (between -1 and $-2 \%$ both for $\mathrm{Ge}_{7}$ and $G e_{7} T$ ). Lower initial values of $R^{*}$ (before the beginning of the compression) suggest a homogeneous coupling between the gels and the TMS, and hence 372 provided limited scope of further improvement during the compression. In this case, the film of water 373 (with an acoustic impedance similar to that of gels) acts as a coupling agent and fills the asperities of the TMS. The results in Figure 5. $b_{1}$ and $5 . b_{2}$ suggest that when a load is applied to the food sample during the compression, the film of water at the interface does not affect the coupling at the interface.

377 (Pramanik et al., 2010), the low and medium levels of lubrication made it possible to observe how the 378 patterns of $\Delta R^{*}$ evolve between dry and high levels. For both levels, $\Delta R^{*}$ first displayed a slight 379 increase, before a more pronounced decrease during the second portion of the compression. The 380 curves related to low and medium levels followed similar trends for most of the compression 381 sequences. It was only at the end of the compression that lower values of $\Delta R^{*}$ were observed for the 382 low level (in a more prominent way for $G_{7} T$ ). Even though the difference of water film thickness 383 between low and medium levels is small, one can notice that it is large enough to detect changes in 384 the US response that are induced by the spreading of the lubricating film at the interface between the food and the TMS. In particular, for the low level, the higher wettability of $G_{7} T$ compared to $G_{7}$ 
(contact angles previously assessed: around $0^{\circ}$ and $40^{\circ}$ respectively (Mantelet et al., 2019)) was likely

387 to promote the spreading of the lubricating film, which may contribute to the explanation of the higher 388 decrease rate of $\Delta R^{*}$ reported for this sample.

\section{Conclusions}

In this study, non-deformable surfaces were considered as TMSs to study the impact of surface

roughness and lubrication on the variations of $R^{*}$ during the compression of different food gels with

different physical properties.

The findings show that both the height and the width of the asperities on the TMSs induces

high variations of the apparent reflection coefficient during the compression of the gels, corresponding suggested that agar dominant products had higher capability for water release or syneresis during the compression. This syneresis phenomenon was observed with the ultrasound method as it led to the the TMSs tended to mitigate the evolution profiles of $\mathrm{R}^{*}$ during a compression.

In the context of food oral processing, the apparent reflection coefficient could help to understand how food gels mold themselves into the surface asperities of the tongue, or how a coupling 406 film of liquid at the interface between the food and the tongue may spread. The method has thus a 407 potential for providing an indirect estimation of the contact area between the tongue surface and 408 food, which would be worthwhile for clarifying the mechanisms underlying sensory perceptions during oral processing (Ares et al., 2007; Szczesniak, 2002). Such an approach could indeed help understanding how the mechanical loads can be transmitted to the mechanoreceptors in the vicinity 
411 of tongue asperities (e.g. in relation with firmness sensations), how the interactions of food and the 412 salivary film with tongue asperities may rule the perceptions of moistness, or also how the fraction of 413 tongue surface in contact with food influence the diffusion of chemical compounds responsible for 414 taste perceptions on tongue surface. our method for such issues. In this view, many physiological issues will need to be accounted for, starting with considering deformable tongue mimicking samples and progressively moving to more realistic motions (involving both compressional and shear motions). All along these improvements, the method should bring new insights for unravelling the determinants of texture perceptions during the manipulation of food between the tongue and the palate.

The shearing of food between the tongue and the palate gives rises to important texture perceptions, particularly for understanding swallowing trigger points. Characterizing the thickness of food samples in tongue-palate tribo-pair is a critical missing information for the identification of the lubrication regime of the system (Rudge et al., 2019). In future work, we could also try to investigate whether an ultrasound method could be used in order to reach this objective.

A longer term perspective could be to extend the development of the method to in vivo applications, with measurements conducted directly on the consumer. Finally, a broader diversity of more realistic food types should be considered (e.g., with irregular shapes and micro or macroscopic structural heterogeneities), and the evolving properties of the food bolus during oral processing should 430 also be examined (e.g., particle size reduction, saliva incorporation). 
434 Debar for their crucial technical support. We also warmly thank Sandrine Mariot for her help with 435 profilometry measurements.

436

\section{Funding}

437

This work was financially supported by (i) the IDI 2015 project funded by IDEX Paris Saclay

438 (ANR-11-IDEX-0003-02), (ii) the PLUS project funded by the French National Institute for Agriculture,

439 Food and Environment (INRAE, 2015), and (iii) the QUSToFood project funded by the French National

440 Research Agency (ANR-17-CE21-004). 
Ares, G., GonçAlvez, D., PéRez, C., ReolóN, G., Segura, N., Lema, P., \& GáMbaro, A. (2007). Influence of gelatin and starch on the instrumental and sensory texture of stirred yogurt. International Journal of Dairy Technology, 60(4), 263-269. https://doi.org/10.1111/j.14710307.2007.00346.x

Awad, T. S., Moharram, H. A., Shaltout, O. E., Asker, D., \& Youssef, M. M. (2012). Applications of ultrasound in analysis, processing and quality control of food: A review. Food Research International, 48(2), 410-427. https://doi.org/10.1016/j.foodres.2012.05.004

Bukowska, M., Essick, G. K., \& Trulsson, M. (2010). Functional properties of low-threshold mechanoreceptive afferent in the human labial mucosa. Experimental Brain Research, 201(1), 59-64. https://doi.org/10.1007/s00221-009-2005-0

Chen, J. (2009). Food oral processing-A review. Food Hydrocolloids, 23(1), 1-25. https://doi.org/10.1016/j.foodhyd.2007.11.013

Chen, J. S., \& Stokes, J. R. (2012). Rheology and tribology: Two distinctive regimes of food texture sensation. Trends in Food Science \& Technology, 25(1), 4-12. https://doi.org/10.1016/j.tifs.2011.11.006

Chen, J.-W., Chang, C.-H., Wang, S.-J., Chang, Y.-T., \& Huang, C.-C. (2014). Submental ultrasound measurment of dynamic tongue base thickness in patients with obstructive sleep apnea. Ultrasound in Medicine \& Biology, 40(11), 2590-2598. http://dx.doi.org/10.1016/j.ultrasmedbio.2014.06.019

Chien, C.-Y., Chen, J.-C., Chang, C.-H., \& Huang, C.-C. (2017). Tracking dynamic tongue motion in ultrasound images for obstructive sleep apnea. Ultrasound in Medicine \& Biology. http://dx.doi.org/10.1016/j.ultrasmedbio.2017.08.001 
465

Clark, A. H., Richardson, R. K., Ross-Murphy, S. B., \& Stubbs, J. M. (1983). Structural and mechanical properties of agar/gelatin co-gels. Small-deformation studies. Macromolecules, 16(8), 13671374.

de Wijk, R. A., Janssen, A. M., \& Prinz, J. F. (2011). Oral movements and the perception of semi-solid $\begin{array}{llll}\text { foods. Physiology } \quad \text { B } & \text { Behavior, }\end{array}$ https://doi.org/10.1016/j.physbeh.2011.04.037

Degrandi-Contraires, É., Beaumont, A., Restagno, F., Weil, R., Poulard, C., \& Léger, L. (2013). CassieWenzel-like transition in patterned soft elastomer adhesive contacts. EPL (Europhysics Letters), 101(1), 14001. https://doi.org/10.1209/0295-5075/101/14001

Foegeding, E. A., Stieger, M., \& van de Velde, F. (2017). Moving from molecules, to structure, to texture perception. Food Hydrocolloids, 68, 31-42. https://doi.org/10.1016/j.foodhyd.2016.11.009

Galén, S., \& Jost-Brinkmann, P.-G. (2010). B-mode and M-mode Ultrasonography of Tongue Movements during Swallowing. Journal of Orofacial Orthopedics / Fortschritte Der Kieferorthopädie, 71(2), 125-135. https://doi.org/10.1007/s00056-010-9928-8

Hiiemae, K. M. (2004). Mechanisms of food reduction, transport and deglutition: how the texture of food affects feeding behavior. Journal of Texture Studies, 35(2), 171-200.

Hsiao, M.-Y., Chang, Y.-C., Chen, W.-S., Chang, H.-Y., \& Wang, T.-G. (2012). Application of ultrasonography in assesing oropharyngeal dysphagia in stroke patients. Ultrasound in Medicine \& Biology, 38(9), 1522-1528. https://doi.org/10.1016/j.ultrasmedbio.2012.04.017

Hutchings, J. B., \& Lillford, P. J. (1988). The Perception of Food Texture - the Philosophy of the Breakdown Path. Journal of Texture Studies, 19(2), 103-115. https://doi.org/10.1111/j.17454603.1988.tb00928.x 
Kim, J.-H., \& Kim, M.-S. (2012). Lateral pharyngeal wall motion analysis using ultrasonography in stroke patients with dysphagia. Ultrasound in Medicine \& Biology, 38(12), 2058-2064. http://dx.doi.org/10.1016/j.ultrasmedbio.2012.07.028

Mantelet, M., Panouille, M., Boue, F., Bosc, V., Restagno, F., Souchon, I., \& Mathieu, V. (2019). Impact of sol-gel transition on the ultrasonic properties of complex model foods: Application to agar/gelatin gels and emulsion filled gels. Food Hydrocolloids, 87, 506-518. https://doi.org/10.1016/j.foodhyd.2018.08.021

Mantelet, M., Restagno, F., Souchon, I., \& Mathieu, V. (2020). Using ultrasound to characterize the tongue-food interface: An in vitro study examining the impact of surface roughness and Iubrication. Ultrasonics, 103, 106095. https://doi.org/10.1016/j.ultras.2020.106095

McEvoy, H., Ross-Murphy, S. B., \& Clark, A. H. (1985). Large deformation and ultimate properties of biopolymer gels: 2. Mixed gel systems. Polymer, 26(10), 1493-1500. https://doi.org/10.1016/0032-3861(85)90082-5

Panouillé, M., Saint-Eve, A., \& Souchon, I. (2016). Instrumental methods for bolus characterization during oral processing to understand food perceptions. Current Opinion in Food Science, 9, 4249. https://doi.org/10.1016/j.cofs.2016.05.002

Peng, C.-L., Jost-Brinkmann, P.-G., Miethke, R.-R., \& Lin, C.-T. (2000). Ultrasonographic measurement of tongue movement during swallowing. Journal of Ultrasound in Medicine, 19(1), 15-20.

Pramanik, R., Osailan, S. M., Challacombe, S. J., Urquhart, D., \& Proctor, G. B. (2010). Protein and mucin retention on oral mucosal surfaces in dry mouth patients. European Journal of Oral Sciences, $118(3), 245-253$.

Rommel, N., \& Hamdy, S. (2016). Oropharyngeal dysphagia: manifestations and diagnosis. Nature $\begin{array}{lllll}\text { Reviews Gastroenterology } \quad \text { Hepatology, } & \text { 13(1), }\end{array}$ https://doi.org/10.1038/nrgastro.2015.199 
511 Santagiuliana, M., Piqueras-Fiszman, B., van der Linden, E., Stieger, M., \& Scholten, E. (2018). Mechanical properties affect detectability of perceived texture contrast in heterogeneous food gels. Food Hydrocolloids, 80, 254-263. https://doi.org/10.1016/j.foodhyd.2018.02.022

514 Shull, K. R. (2002). Contact mechanics and the adhesion of soft solids. Materials Science and Engineering: R: Reports, 36(1), 1-45. https://doi.org/10.1016/S0927-796X(01)00039-0

516 Stokes, J. R., Boehm, M. W., \& Baier, S. K. (2013). Oral processing, texture and mouthfeel: From rheology to tribology and beyond. Current Opinion in Colloid \& Interface Science, 18(4), 349359. https://doi.org/10.1016/j.cocis.2013.04.010

Stone, M. (2005). A guide to analysing tongue motion from ultrasound images. Clinical Linguistics \& Phonetics, 19(6-7), 455-501. https://doi.org/10.1080/02699200500113558

Szczesniak, A. S. (2002). Texture is a sensory property. Food Quality and Preference, 13(4), 215-225.

Uemori, N., Kakinoki, Y., Karaki, J., \& Kakigawa, H. (2012). New method for determining surface 523 roughness of tongue. Gerodontology, 29(2), 90-95. https://doi.org/10.1111/j.17412358.2011.00509.x

Weng, C.-K., Chien, J.-W., Lee, P.-Y., \& Huang, C.-C. (2017). Implementation of a wearable ultrasound device for the overnight monitoring of tongue base deformation during obstructive sleep apnea events. Ultrasound in Medicine \& Biology, 43(8), 1639-1650. http://dx.doi.org/10.1016/j.ultrasmedbio.2017.04.004 

to yellow).

Figure 2: (a) Means and standard deviations of $R^{*}$ before the beginning of the compression $(\varepsilon=0)$ of three types of gels $\left(\left(a_{1}\right) G e_{7},\left(a_{2}\right) G e_{3.5}\right.$ and $\left.\left(a_{3}\right) A g_{0.3}\right)$ on the different TMSs. (b) Variations of $\Delta R^{*}$ during the compression of three types of gels $\left(\left(b_{1}\right) G e_{7},\left(b_{2}\right) G e_{3.5}\right.$ and $\left.\left(b_{3}\right) A g_{0.3}\right)$ on the different TMSs. The results were obtained under dry lubrication conditions. variations of $R^{*}$ before the beginning of the compression as a function of the Young's modulus (error bars corresponding to standard deviations and a broken line draws the linear regression for indicative 545 purpose). The results were obtained with $R_{1} B_{1}$ under dry lubrication conditions. before the beginning of the compression as a function of the Young's modulus (error bars corresponding to standard deviations and a broken line draws the linear regression for indicative purpose). The results were obtained with $R_{1} B_{1}$ under dry lubrication conditions. 
558 deviation).

\begin{tabular}{|c|c|c|c|c|c|c|}
\hline \multirow{4}{*}{ Gel } & \multirow{4}{*}{$\begin{array}{l}\text { Dominant } \\
\text { Polymer }\end{array}$} & \multicolumn{4}{|c|}{ Composition (wt\%) } & \multirow{4}{*}{$\begin{array}{c}\text { Young's modulus } \\
\text { (kPa) }\end{array}$} \\
\hline & & \multirow{3}{*}{ Water } & \multirow{3}{*}{ Agar } & \multirow{3}{*}{ Gelatin } & \multirow{3}{*}{$\begin{array}{c}\text { TWEEN } \\
20\end{array}$} & \\
\hline & & & & & & \\
\hline & & & & & & \\
\hline$A g_{0.3}$ & Agar & 84.7 & 0.3 & - & - & $4.2 \pm 3.2$ \\
\hline$A g_{1} G e_{0.75}$ & Agar & 84.25 & 1 & 0.75 & - & $58.4 \pm 14.7$ \\
\hline$A g_{1.8}$ & Agar & 83.3 & 1.8 & - & - & $132.0 \pm 9.7$ \\
\hline$G e_{3.5}$ & Gelatin & 81.5 & - & 3.5 & - & $2.2 \pm 0.4$ \\
\hline$A g_{0,3} G_{3,5}$ & Gelatin & 81.2 & 0.3 & 3.5 & - & $12.7 \pm 2.9$ \\
\hline$A g_{0,7} G e_{5.85}$ & Gelatin & 78.45 & 0.7 & 5.85 & - & $32.1 \pm 7.2$ \\
\hline $\mathrm{Ge}_{7}$ & Gelatin & 78 & - & 7 & - & $24.0 \pm 2.2$ \\
\hline $\mathrm{Ge}_{7} \mathrm{~T}$ & Gelatin & 77.25 & - & 7 & 1.5 & $17.2 \pm 1.8$ \\
\hline
\end{tabular}

559

560

561

562

563 

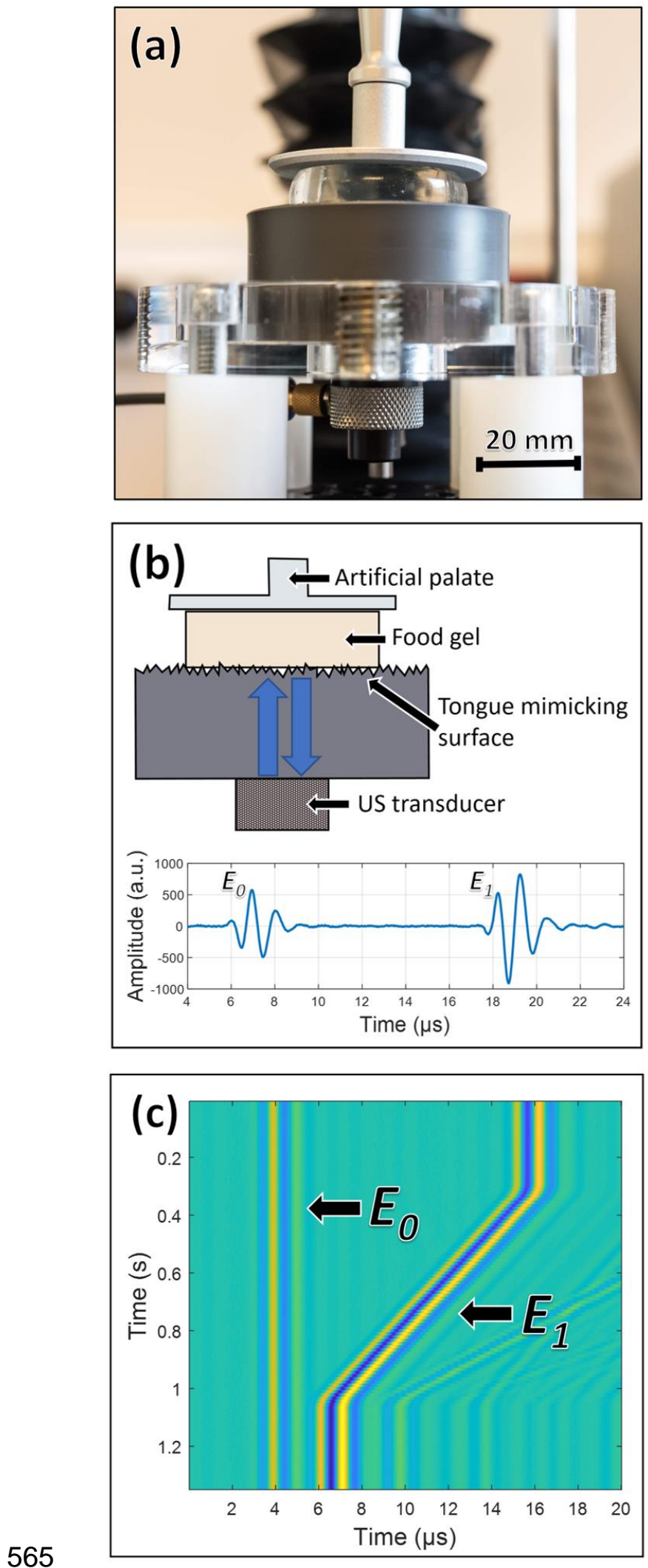

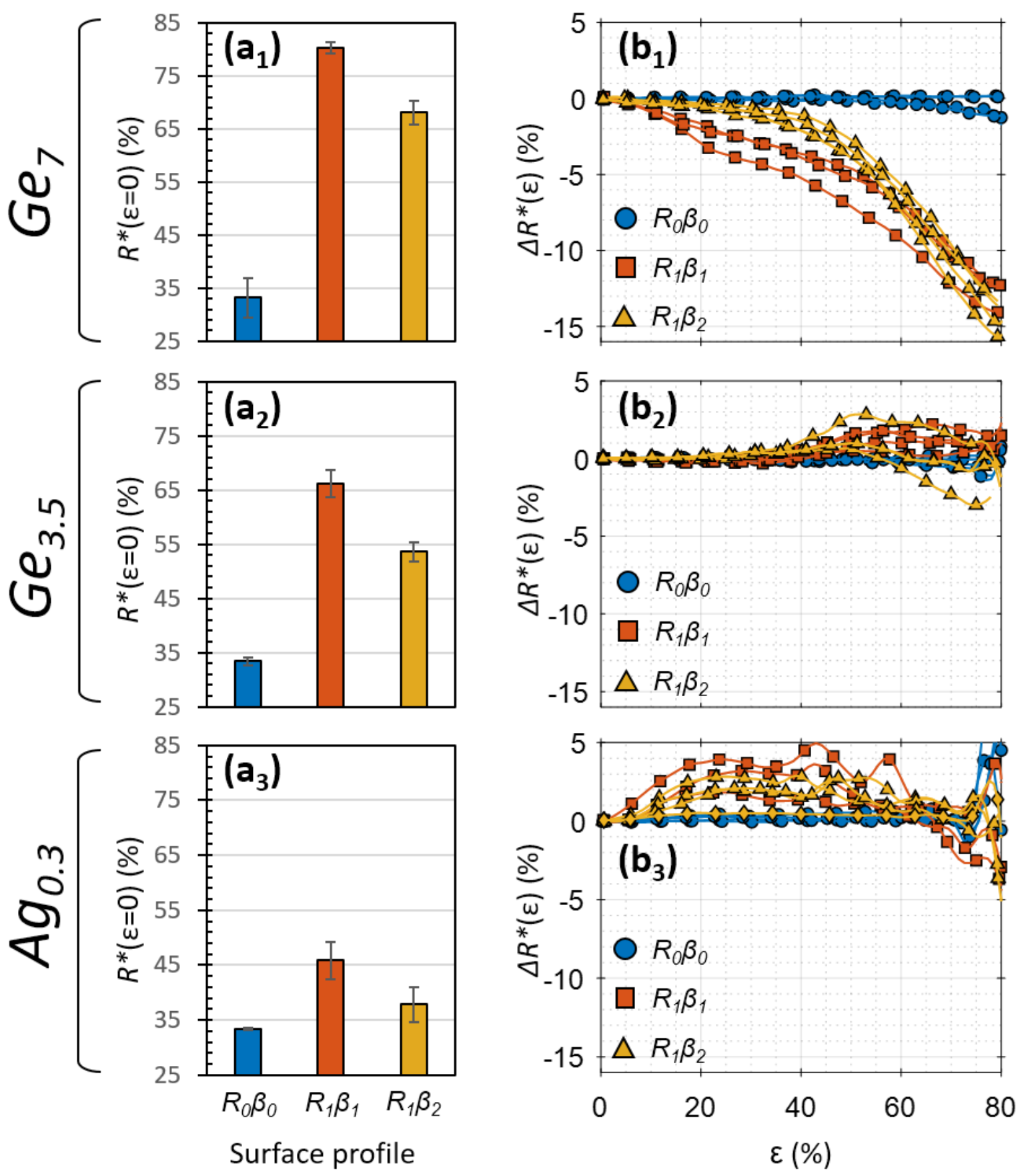
Figure 3
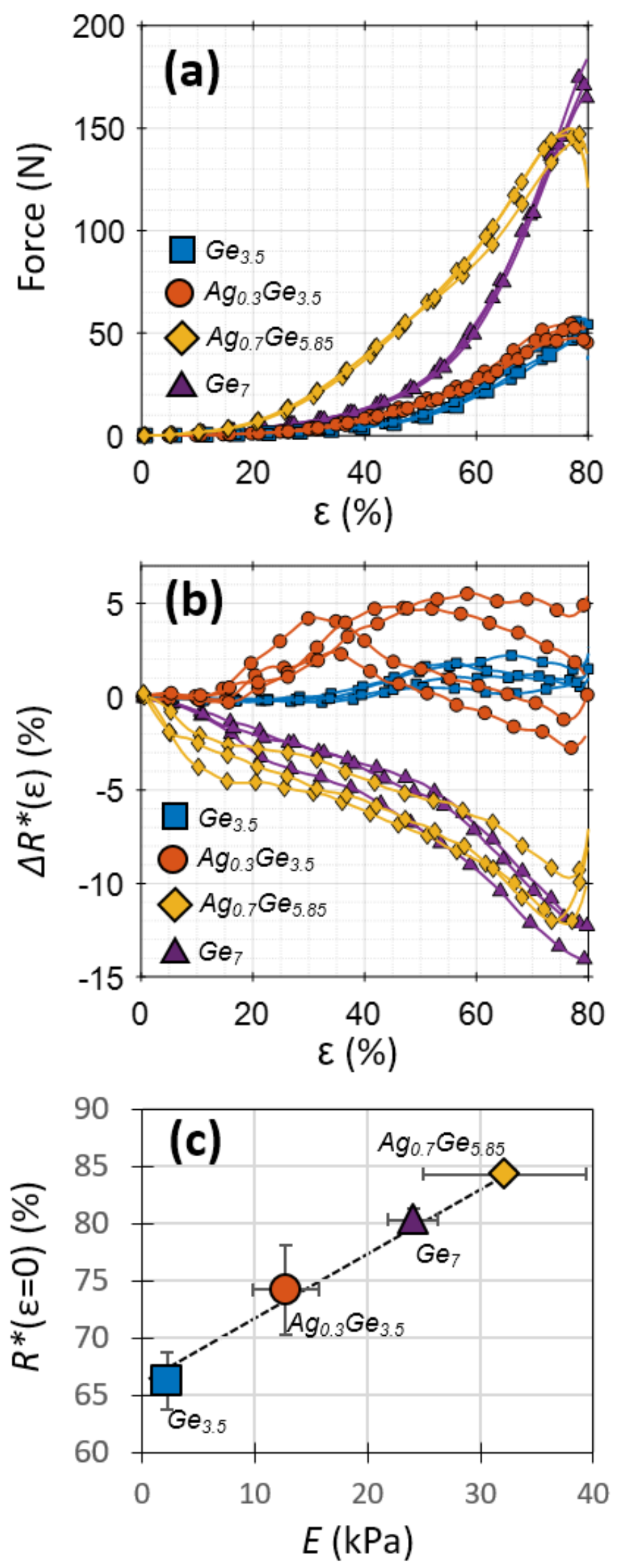

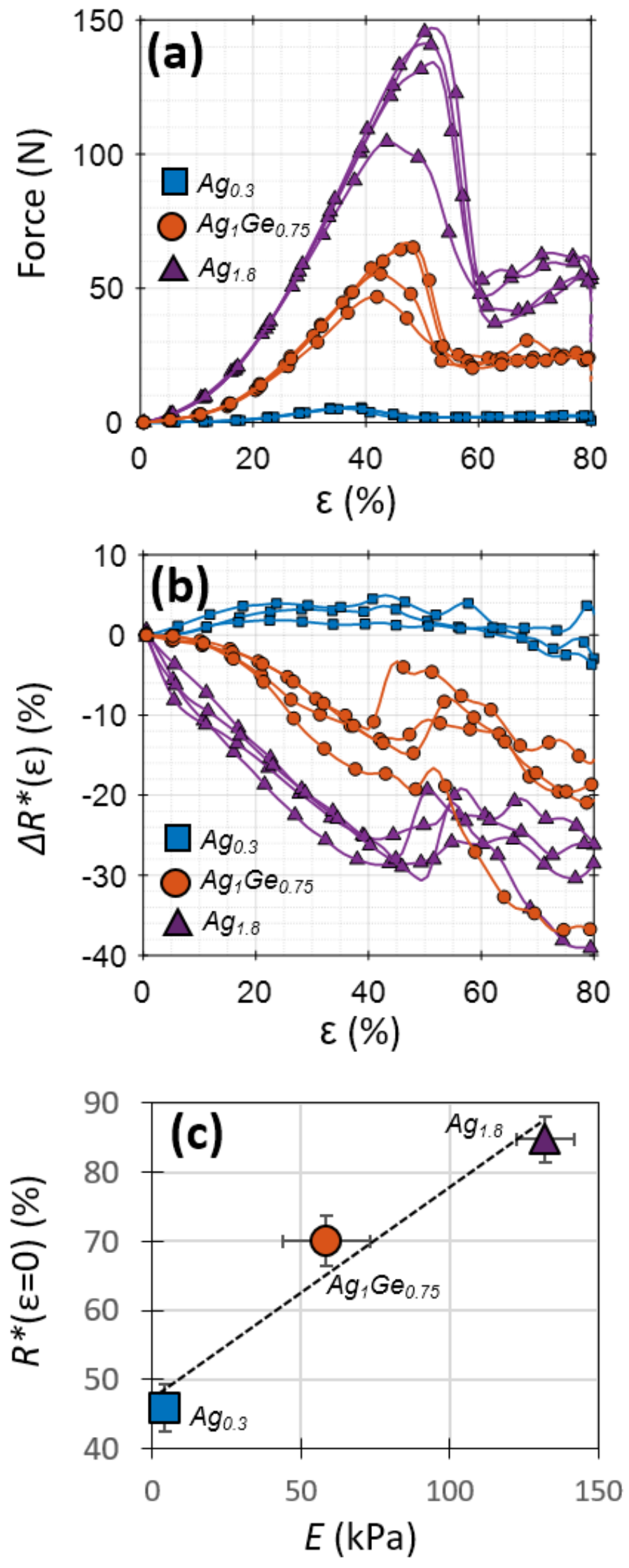

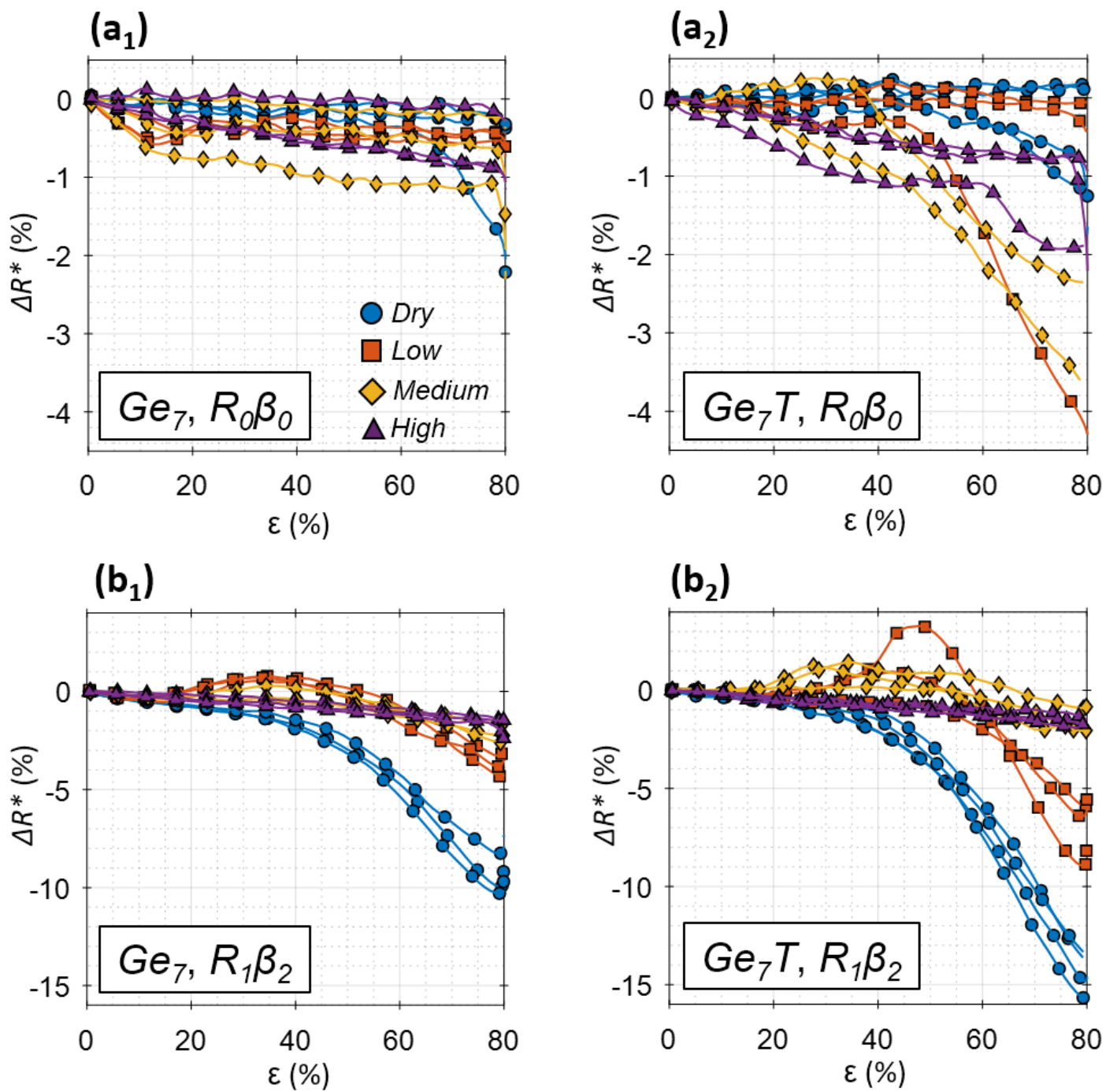